\title{
Technique matters
}

\author{
Peter T Scardino
}

Against a historical yardstick, surgery as a scientific discipline is fairly new. Although basic surgical techniques have existed for centuries, routinely incising into the anesthetized human body, and manipulating its organs and tissues for the benefit of the ill, would seem miraculous to someone born two centuries ago. Widespread standards for surgical residency training, and intense regulation by medical organizations and governments around the world, have reassured the public that surgery is generally safe.

Systems of national education, training, certification and credentialing have proven effective in guaranteeing minimum standards for surgical procedures in many countries. But can we do better? Are the differences in outcomes of major surgical procedures related simply to patient selection or do differences in technique and perioperative care matter?

Health services research has addressed this question by examining how perioperative morbidity varies by hospital and surgeon volume. These variations point to underlying differences in quality of care. The methodology comes from the field of industrial engineering, which applies statistical analysis to document variations in each step of the process of production, substantially improving the quality of the final product.

There are clear differences in outcomes among surgeons performing major urological procedures. In the US, operative mortality after cystectomy is almost twice as high in the hands of low- compared to high-volume surgeons, a difference of 2.4 deaths for every 100 cystectomies. ${ }^{1}$ Mortality after nephrectomy for cancer was significantly higher in the lowest volume hospitals than in the highest. ${ }^{2}$ Radical prostatectomy perioperative complication rates are significantly lower when performed by high-volume surgeons. ${ }^{3,4}$ Volume is not the only factor: perioperative complications, anastomotic strictures, and long-term incontinence rates vary markedly, even among highvolume surgeons. Furthermore, surgeons with
Are the

differences in outcomes of major surgical procedures related simply to patient selection or do differences in surgical technique and perioperative care matter?

PT Scardino is the Editor-in-Chief of Nature Clinical Practice Urology, Chairman of the Department of Urology at Sidney Kimmel Center for Prostate and Urologic Cancers, and Alfred $P$ Sloan Chair at Memorial Sloan-Kettering Cancer Center.

\section{Competing interests}

The author declared he has no competing interests.

www.nature.com/clinicalpractice doi:10.1038/ncpuro0058 poor outcomes by one measure are more likely to have poor outcomes by the other two. ${ }^{4}$

Society has a clear interest in improved surgical outcomes. In the US, the national Surgical Care Improvement Project (www.medqic. org) has established evidence-based strategies to improve surgical quality. Their goal is to reduce major perioperative complications by $25 \%$ before 2010 . The Leapfrog Group (www.leapfroggroup.org) rates hospitals by quality, encourages public reporting of outcomes, and steers patients to high-volume hospitals for complex procedures.

A good outcome depends more on fundamental factors such as patient selection, anesthesia, perioperative care, surgical technique and sound judgment, than volume. All of these factors can be more readily assessed in high-volume settings.

The introduction of laparoscopic surgery has focused attention on surgical technique for the first time in decades. The field of outcomes research provides tools for measuring results comprehensively and objectively. In the future we are likely to see many more studies of the quality of surgical care and clinical trials directly comparing the outcomes of different procedures. How should we respond to measured differences in outcomes?

Steerage of patients to high-volume providers will grow, but is likely to have less effect on rural than urban centers. Specialization will continue, in private practice as well as in university centers, as it offers competitive advantages as well as improved outcomes. Education can also play a critical role. An effective educational program can substitute for years of experience. Accepting the facts is the first step: surgical technique matters. Surgeons can learn to improve, if they want to, and if high-quality educational programs are available from their professional organizations.

Supplementary information, in the form of a reference list, is available on the Nature Clinical Practice Urology website. 